\title{
HLA-A2-Binding TYR/MART-1/gp100 Multipeptide-Pulsed Autologous Dendritic Cell Vaccine
}

National Cancer Institute

\section{Source}

National Cancer Institute. HLA-A2-Binding TYR/MART-1/gp100 Multipeptide-Pulsed

Autologous Dendritic Cell Vaccine. NCI Thesaurus. Code C90569.

A cell-based cancer vaccine composed of autologous dendritic cells (DCs) pulsed with human leukocyte antigen (HLA)-A2-restricted melanoma-associated antigen peptides tyrosinase (TYR), MART-1 (melanoma antigen recognized by T-cells) and melanoma antigen glycoprotein 100 (gp100), with potential immunomodulating and antineoplastic activity. Upon vaccination, HLA-A2-binding TYR/MART-1/gp100 multipeptide-pulsed autologous dendritic cell vaccine may stimulate the host immune system to mount an anti-tumoral cytotoxic T lymphocyte (CT L) and antibody responses ag ainst Tyr-, MART -1 and gp100-expressing cancer cells, resulting in tumor cell lysis. HLA-A2 is an MHC class I molecule that presents antigenic peptides to CD8+ T cells; epitope design restricted to epitopes that bind most efficiently to HLA-A2 may improve antigenic peptide immunogenicity. 